\title{
Acoustic Wave Propagation in a Composite of Two Different Poroelastic Materials with a Very Rough Periodic Interface: a Homogenization Approach
}

\author{
Robert P. Gilbert \\ Department of Mathematical Sciences \\ University of Delaware, Newark, DE 19716, U.S.A. \\ Miao-jung $\mathrm{Ou}$ \\ Institute for Mathematics and its Applications, \\ University of Minnesota, Minneapolis, MN 55455, USA
}

October 18, 2002

\begin{abstract}
Homogenization is used to analyze the system of Biot-type partial differential equations in a domain of two different poroelastic materials with a very rough periodic interface. It is shown that by using homogenization, such a rough interface can be replaced by an equivalent flat layer within which a system of modified differential equations holds. The coefficients of this new system of equations are certain "effective" parameters. These coefficients are determined by solutions of the auxiliary problems which involve the detailed structure of the interface. In this paper, the auxiliary problems are derived and the homogenized system of equations is given.
\end{abstract}

\section{Introduction}

Poroelasticity, the mechanics of porous elastic solids with fluid-filled pores, has received attention in the last few decades for its important role in oil recovery, the study of the triggering of earthquakes, liquid waste disposed by underground seepage into pores, and underwater acoustics involving propagation in the water-saturated, porous bottom of the ocean, etc.

In underwater acoustics, a poroelastic seabed model is more realistic than a rigid seabed or an elastic seabed. In each of these three models, the sea bottom and the interface between different layers are usually assumed to be flat. In [2], acoustic wave propagation in a shallow ocean with a flat poroelastic seabed was studied. Here in contrast, we take into account the geometry of the interface and study its effect on acoustic wave propagation. We will assume the interface between the two poroelastic layers to be very rough. For simplicity, we assume that the interface has a periodic geometry, i.e. the ratio of the amplitude to the "wavelength" is large.

Problems involving rough boundaries or interfaces can usually be analyzed using perturbation methods when the amplitude/wavelength ratio is small. For problems with a large ratio, other methods of analysis are required. For example, the homogenization method used by Kohler, Papanicolaou and Varadhan [3], or Nevard and Keller [4]. In their works, it is shown that for certain partial differential equations, the problem of the PDE in the region which contains the rough interface can be replaced by another problem of a homogenized PDE in an equivalent flat layer. The basic assumption underlying the method is that the scale length, or "wavelength" $\epsilon$ of the roughness is small compared with all other relevant lengths, especially the roughness amplitude. 
In this paper, we use a homogenization method, similar to the one mentioned above, to study the timeharmonic acoustic wave propagation in a composite of two different poroelastic materials. The auxiliary problems and the homogenized equations in the equivalent layer will be derived.

\section{The Constitutive Equations}

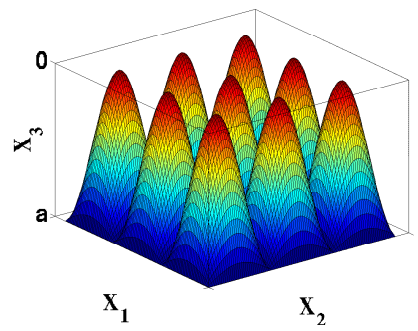

Figure 1: Schematic representation of the rough interface

The poroelastic equations derived by Biot $[5,6,7]$ have long been regarded as standard in solving problems in poroelasticity. However, the validity of these equations has recently been questioned. For this reason, various authors have used homogenization methods to derive anew the governing equations of linear poroelasticity by starting with the detailed micro-structure of the pores. For example, Burridge and Keller [8], Gilbert and Mikelić [9], Auriault [10], and Auriault et al. [11]. In these papers, the pores are assumed to have periodic structure, and the linearized equations of elasticity and the linearized Navier-Stokes equations are used to describe the behavior of the solid part and the fluid part, respectively. These works have shown that the newly derived equations coincide with Biot's equations when the dimensionless viscosity of the fluid is small. Furthermore, their works also enable us to perform a complete calculation of the effective parameters in Biot's equations.

Since the pore fluid in the seabed can be regarded as Newtonian and incompressible, we will adapt the equations given by Auriault et al. in [11]. For time harmonic motion $\tilde{u}(\mathbf{x}, t)=u(\mathbf{x}) e^{i \omega t}, \tilde{p}(\mathbf{x}, t)=p(\mathbf{x}) e^{i \omega t}$ in an isotropic poroelastic medium, they are

$$
\begin{aligned}
\boldsymbol{\Sigma} & =\boldsymbol{C e}(\boldsymbol{u})-\tilde{\alpha} p \boldsymbol{I}, \\
\operatorname{div}(\boldsymbol{\Sigma}) & =-\omega^{2}\left\{\rho_{s}(1-f) \boldsymbol{u}+f \rho_{l} \boldsymbol{U}_{l}\right\}, \\
\mathcal{I} f \omega\left(\boldsymbol{U}_{l}-\boldsymbol{u}\right) & =\boldsymbol{K}\left(\omega^{2} \rho_{l} \boldsymbol{u}-\operatorname{grad} p\right), \\
f \operatorname{div}\left(\boldsymbol{U}_{l}-\boldsymbol{u}\right) & =-\tilde{\alpha} \operatorname{div} \boldsymbol{u}-\tilde{\beta} p .
\end{aligned}
$$

In these equations, $\boldsymbol{I}$ is the unit tensor and $\mathcal{I}=\sqrt{-1} . \boldsymbol{\Sigma}$ represents the total stress tensor; $\boldsymbol{C}$ is the elasticity tensor of the skeleton, $p$ the fluid pressure ( positive for compression), $\omega$ the acoustic frequency, $\boldsymbol{u}$ the displacement of the solid part, $\boldsymbol{U}_{l}$ the displacement of the fluid part, $\boldsymbol{e}(\boldsymbol{u})$ the strain tensor, $f$ the porosity, $\rho_{l}$ the density of the pore fluid, $\rho_{s}$ the density of the skeleton and $\boldsymbol{K}$ is the generalized Darcy permeability tensor, which is introduced by the homogenization theory, symmetric and $\omega$-dependent. The effective parameters $\tilde{\alpha}$ and $\tilde{\beta}$ can be computed. 
In [12], using Helmholtz's decomposition theorem, it is shown that the degree of freedom of (1)-(4) is only four. Therefore, we may reformulate this system in terms of $\boldsymbol{u}$ and $p$. This gives (5) and (6). The interface between the two different poroelastic materials is described by $x_{3}=h\left(x_{1}, x_{2}\right)$, where $h$ is $\epsilon$-periodic in both the $x_{1}$ and $x_{2}$ direction ( See Fig. 1). The physical parameters are assumed to be constants above and below the interface and have a jump discontinuity across the interface. In what follows, we adopt the summation convention, Latin subscripts taking the values 1,2, 3 and Greek subscripts taking the values 1,2.

$$
\begin{aligned}
\partial_{x_{j}}\left(C_{i j k l} \partial_{x_{l}} u_{k}\right)-\tilde{\alpha} \delta_{i j} p+\omega^{2}\left(\breve{\rho}_{i j} u_{j}\right) & =-\breve{\alpha}_{i j} \partial_{x_{j}} p, x_{3} \neq h\left(x_{1}, x_{2}\right), \\
-\frac{\mathcal{I}}{\omega} K_{i j}\left(\partial_{x_{i}} \partial_{x_{j}} p-\rho_{l} \omega^{2} \partial_{x_{i}} u_{j}\right)-\tilde{\alpha} \partial_{x_{i}} u_{i} & =\tilde{\beta} p, x_{3} \neq h\left(x_{1}, x_{2}\right), \\
{[\boldsymbol{u}] } & =0 \\
{\left[n_{j}\left(C_{i j k l} \partial_{x_{l}} u_{k}-\tilde{\alpha} \delta_{i j} p\right)\right] } & =0 \\
{[p] } & =0 \\
{\left[n_{i} K_{i j}\left(\partial_{x_{j}} p-\rho_{l} \omega^{2} \partial_{x_{i}} u_{j}\right)\right] } & =0
\end{aligned}
$$

where

$$
\begin{aligned}
\breve{\alpha}_{i j} & :=\mathcal{I} \omega \rho_{l} K_{i j}, \\
\breve{\rho}_{i j} & :=\rho_{s}(1-f) \delta_{i j}+\rho_{l}\left\{-\breve{\alpha}_{i j}+f \delta_{i j}\right\}
\end{aligned}
$$

and $[\cdot]$ denotes the jump across the interface $x_{3}=h\left(x_{1}, x_{2}\right)$, i.e.

$$
\left[F\left(\boldsymbol{x}^{*}\right)\right]:=\lim _{Y^{-} \ni \boldsymbol{x} \rightarrow \boldsymbol{x}^{*} \in \Gamma} F(\boldsymbol{x})-\lim _{Y^{+} \ni \boldsymbol{x} \rightarrow \boldsymbol{x}^{*} \in \Gamma} F(\boldsymbol{x}) .
$$

In (5)-(10), $\boldsymbol{\delta}$ is the Kronecker delta tensor and $\boldsymbol{n}$ is the unit normal vector of the interface $x_{3}=h\left(x_{1}, x_{2}\right)$

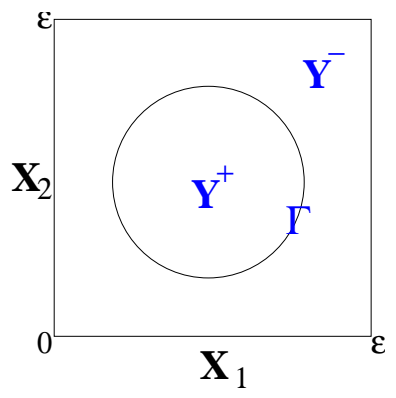

Figure 2: Schematic representation of the $x_{1} x_{2}$ profile of the periodicity cell $Y$.

pointing in the positive $x_{3}$ direction. The interface conditions (7), (8), (9) and (10) represent continuity of displacement, normal stress, pressure and the total velocity across the interface, respectively.

\section{Formal Expansions and the Homogenized Equations}

We introduce a new independent variable $\boldsymbol{y}$, where $y_{\alpha}:=\frac{x_{\alpha}}{\epsilon}, \alpha=1,2$. Using the fact that $\boldsymbol{n}$ is proportional to $\left(\epsilon^{-1} \partial_{y_{1}} h, \epsilon^{-1} \partial_{y_{2}} h,-1\right),(8)$ and (10) become

$$
\begin{aligned}
& {\left[\epsilon^{-1}\left(\partial_{y_{\beta}} h\right)\left(C_{i \beta k l} \partial_{x_{l}} u_{k}-\tilde{\alpha} p \delta_{i \beta}\right)-\left(C_{i 3 k l} \partial_{x_{l}} u_{k}-\tilde{\alpha} p \delta_{i 3}\right)\right]=0,} \\
& {\left[\epsilon^{-1} K_{\alpha j}\left(\partial_{x_{j}} p-\rho_{l} \omega^{2} u_{j}\right)\left(\partial_{y_{\alpha}} h\right)-K_{3 j}\left(\partial_{x_{j}} p-\rho_{l} \omega^{2} u_{j}\right)\right]=0 .}
\end{aligned}
$$


Define new dependent variables $w_{i}$ and $q$ such that

$$
\begin{aligned}
w_{i}(\boldsymbol{x}, \boldsymbol{y}, t, \epsilon) & :=u_{i}(\boldsymbol{x}, t, \epsilon), \\
q(\boldsymbol{x}, \boldsymbol{y}, t, \epsilon) & :=p(\boldsymbol{x}, t, \epsilon) .
\end{aligned}
$$

Note that $\partial_{x_{\alpha}} u_{i}=\left(\partial_{x_{\alpha}}+\epsilon^{-1} \partial_{y_{\alpha}}\right) w_{i}$ and $\partial_{x_{\alpha}} p=\left(\partial_{x_{\alpha}}+\epsilon^{-1} \partial_{y_{\alpha}}\right) q$.

In terms of the new variables, (5) and (6) can be written as

$$
\begin{aligned}
& \epsilon^{-2} \partial y_{\beta}\left(C_{i \beta k \delta} \partial_{y_{\delta}} w_{k}\right) \\
+ & \epsilon^{-1}\left\{\partial_{x_{j}}\left(C_{i j k \delta} \partial_{y_{\delta}} w_{k}\right)+\partial_{y_{\beta}}\left(C_{i \beta k l} \partial_{x_{l}} w_{k}\right)+\breve{\alpha}_{i \gamma} \partial_{y_{\gamma}} q-\tilde{\alpha} \delta_{i \gamma} \partial_{y_{\gamma}} q\right\} \\
+ & \epsilon^{0}\left\{\partial_{x_{j}}\left(C_{i j k l} \partial_{x_{l}} w_{k}\right)+\omega^{2} \breve{\rho}_{i j} w_{j}+\breve{\alpha}_{i j} \partial_{x_{j}} q-\tilde{\alpha} \delta_{i j} \partial_{x_{j}} q\right\}=0, \\
& \epsilon^{-2}\left(-\frac{\mathcal{I}}{\omega} K_{\alpha \beta} \partial_{y_{\alpha}} \partial_{y_{\beta}} q\right) \\
+ & \epsilon^{-1}\left\{\mathcal{I} \omega \rho_{l} K_{\alpha j} \partial_{y_{\alpha}} w_{j}-\tilde{\alpha} \partial_{y_{\alpha}} w_{\alpha}-\frac{2 \mathcal{I}}{\omega} K_{\alpha j} \partial_{x_{j}} \partial_{y_{\alpha}} q\right\} \\
+ & \epsilon^{0}\left\{\mathcal{I} \omega \rho_{l} K_{i j} \partial_{x_{i}} w_{j}-\frac{\mathcal{I}}{\omega} K_{i j} \partial_{x_{i}} \partial_{x_{j}} q-\tilde{\alpha} \partial_{x_{i}} w_{i}-\tilde{\beta} q\right\}=0 .
\end{aligned}
$$

Now we assume that $\boldsymbol{w}$ and $q$ have the following asymptotic expansions for $\epsilon$ small:

$$
\begin{aligned}
\boldsymbol{w}(\boldsymbol{x}, \boldsymbol{y}, t, \epsilon) & =\boldsymbol{u}^{(0)}(\boldsymbol{x}, \boldsymbol{y}, t)+\sum_{k=1}^{2} \boldsymbol{u}^{(k)}(\boldsymbol{x}, \boldsymbol{y}, t) \epsilon^{k}+\mathcal{O}\left(\epsilon^{3}\right) \\
q(\boldsymbol{x}, \boldsymbol{y}, t, \epsilon) & =p^{(0)}(\boldsymbol{x}, \boldsymbol{y}, t)+\sum_{k=1}^{2} p^{(k)}(\boldsymbol{x}, \boldsymbol{y}, t) \epsilon^{k}+\mathcal{O}\left(\epsilon^{3}\right)
\end{aligned}
$$

We also assume that each term in the expansions is $Y$-periodic and that the asymptotic forms of the derivatives of $\boldsymbol{w}$ and $q$ are given by term by term differentiation of (15) and (16), respectively. By equating the terms of the same order in $\epsilon$, we get the following equations:

$\mathcal{O}\left(\epsilon^{-2}\right):$

$$
\begin{aligned}
& \partial_{y_{\beta}}\left(C_{i \beta k \delta} \partial_{y_{\delta}} u_{k}^{(0)}\right)=0, \\
& K_{\alpha \beta} \partial_{y_{\alpha}} \partial_{y_{\beta}} p^{(0)}=0, \\
& {\left[C_{i \beta k \delta} \partial_{y_{\delta}} u_{k}^{(0)} \partial_{y_{\beta}} h\right]=0} \\
& {\left[K_{\alpha \beta} \partial_{y_{\beta}} p^{(0)} \partial_{y_{\alpha}} h\right]=0 .}
\end{aligned}
$$


$\mathcal{O}\left(\epsilon^{-1}\right):$

$$
\begin{aligned}
& \partial_{y_{\beta}}\left(C_{i \beta k \delta} \partial_{y_{\delta}} u_{k}^{(1)}\right)+\partial_{x_{j}}\left(C_{i j k \delta} \partial_{y_{\delta}} u_{k}^{(0)}\right)+\partial_{y_{\beta}}\left(C_{i \beta k l} \partial_{x_{l}} u_{k}^{(0)}\right) \\
& +\breve{\alpha}_{i \gamma} \partial_{y_{\gamma}} p^{(0)}-\tilde{\alpha} \delta_{i \gamma} \partial_{y_{\gamma}} p^{(0)}=0, \\
- & \frac{\mathcal{I}}{\omega} K_{\alpha \beta} \partial_{y_{\alpha}} \partial_{y_{\beta}} p^{(1)}+\mathcal{I} \omega \rho_{l} K_{\alpha j} \partial_{y_{\alpha}} u_{j}^{(0)}-\tilde{\alpha} \partial_{y_{\alpha}} u_{\alpha}^{(0)} \\
- & \frac{2 \mathcal{I}}{\omega} K_{\alpha j} \partial_{y_{\alpha}} \partial_{x_{j}} p^{(0)}=0 \\
& {\left[\left(C_{i \beta k \delta} \partial_{y_{\delta}} u_{k}^{(1)}+C_{i \beta k l} \partial_{x_{l}} u_{k}^{(0)}-\tilde{\alpha} p^{(0)} \delta_{i \beta}\right)\left(\partial_{y_{\beta}} h\right)-C_{i 3 k \delta} \partial_{y_{\delta}} u_{k}^{(0)}\right]=0, } \\
& {\left[\left(K_{\alpha \beta} \partial_{y_{\beta}} p^{(1)}+K_{\alpha j}\left(\partial_{x_{j}} p^{(0)}-\rho_{l} \omega^{2} u_{j}^{(0)}\right)\right)\left(\partial_{y_{\alpha}} h\right)-K_{3 \beta} \partial_{y_{\beta}} p^{(0)}\right]=0 . }
\end{aligned}
$$

$\mathcal{O}\left(\epsilon^{0}\right)$ :

$$
\begin{aligned}
& \partial_{y_{\beta}}\left(C_{i \beta k \delta} \partial_{y_{\delta}} u_{k}^{(2)}\right)+\partial_{x_{j}}\left(C_{i j k \delta} \partial_{y_{\delta}} u_{k}^{(1)}\right)+\partial_{y_{\beta}}\left(C_{i \beta k l} \partial_{x_{l}} u_{k}^{(1)}\right) \\
& +\breve{\alpha}_{i \gamma} \partial_{y_{\gamma}} p^{(1)}-\tilde{\alpha} \delta_{i \gamma} \partial_{y_{\gamma}} p^{(1)}+\partial_{x_{j}}\left(C_{i j k l} \partial_{x_{l}} u_{k}^{(0)}\right) \\
& +\omega^{2} \breve{\rho}_{i j} u_{j}^{(0)}+\breve{\alpha}_{i j} \partial_{x_{j}} p^{(0)}-\tilde{\alpha} \delta_{i j} \partial_{x_{j}} p^{(0)}=0 \\
& -\frac{\mathcal{I}}{\omega} K_{\alpha \beta} \partial_{y_{\alpha}} \partial_{y_{\beta}} p^{(2)}+\mathcal{I} \omega \rho_{l} K_{\alpha j} \partial_{y_{\alpha}} u_{j}^{(1)}-\tilde{\alpha} \partial_{y_{\beta}} u_{\beta}^{(1)}-\frac{2 \mathcal{I}}{\omega} K_{\alpha j} \partial_{x_{j}} \partial_{y_{\alpha}} p^{(1)} \\
& +\mathcal{I} \omega \rho_{l} K_{i j} \partial_{x_{i}} u_{j}^{(0)}-\tilde{\alpha} \partial_{x_{i}} u_{i}^{(0)}-\frac{\mathcal{I}}{\omega} K_{i j} \partial_{x_{i}} \partial_{x_{j}} p^{(0)}-\tilde{\beta} p^{(0)}=0, \\
& {\left[\left(C_{i \beta k \delta} \partial_{y_{\delta}} u_{k}^{(2)}+C_{i \beta k l} \partial_{x_{l}} u_{k}^{(1)}-\tilde{\alpha} p^{(1)} \delta_{i \beta}\right)\left(\partial_{y_{\beta}} h\right)\right.} \\
& \left.-C_{i 3 k \delta} \partial_{y_{\delta}} u_{k}^{(1)}-C_{i 3 k l} \partial_{x_{l}} u_{k}^{(0)}+\tilde{\alpha} p^{(0)} \delta_{i 3}\right]=0, \\
& {\left[\left(K_{\alpha \beta} \partial_{y_{\beta}} p^{(2)}+K_{\alpha j}\left(\partial_{x_{j}} p^{(1)}-\rho_{l} \omega^{2} u_{j}^{(1)}\right)\right)\left(\partial_{y_{\alpha}} h\right)\right.} \\
& \left.-K_{3 \beta} \partial_{y_{\beta}} p^{(1)}-K_{3 j}\left(\partial_{x_{j}} p^{(0)}-\rho_{l} \omega^{2} u_{j}^{(0)}\right)\right]=0 \\
& {\left[\boldsymbol{u}^{(0)}\right]=0} \\
& {\left[p^{(0)}\right]=0}
\end{aligned}
$$

We now consider equations (17), (19) and (29), which correspond to $\boldsymbol{u}^{(0)}$ :

$$
\begin{aligned}
& \partial_{y_{\beta}}\left(C_{i \beta k \delta} \partial_{y_{\delta}} u_{k}^{(0)}\right)=0, \\
& {\left[C_{i \beta k \delta} \partial_{y_{\delta}} u_{k}^{(0)} \partial_{y_{\beta}} h\right]=0,} \\
& {\left[u^{(0)}\right]=0 .}
\end{aligned}
$$

By scalar multiplying (31) by $\overline{\boldsymbol{u}^{(0)}}$, then integrating the product over the periodicity cell $Y$ ( see Figure 2 ) 
and applying the $Y$-periodic condition to $u^{(0)}$, we have

$$
\begin{aligned}
& \int_{Y} C_{i \beta k \delta} \partial_{y_{\delta}} u_{k}^{(0)} \overline{\partial_{y_{\beta}} u_{i}^{(0)}} d \boldsymbol{y} \\
= & -\int_{\Gamma}\left[\overline{u_{i}^{(0)}} C_{i \beta k \delta} \partial_{y_{\delta}} u_{k}^{(0)} \partial_{y_{\beta}} h\right] \frac{d \sigma}{\left|\nabla_{\boldsymbol{y}} h\right|},
\end{aligned}
$$

where

$$
\nabla_{\boldsymbol{y}} h:=\left(\partial_{y_{1}} h, \partial_{y_{2}} h\right)^{T} .
$$

We apply the jump conditions (32) and (33) to (34) to obtain

$$
\int_{Y} C_{i \beta k \delta} \partial_{y_{\delta}} u_{k}^{(0)} \overline{\partial_{y_{\beta}} u_{i}^{(0)}} d \boldsymbol{y}=0 .
$$

By the symmetry of $C_{i j k l}$ and $\partial_{y_{\beta}} u_{i}^{(0)}$, we have

$$
\partial_{y_{\beta}} u_{i}^{(0)}=0
$$

i.e. $\boldsymbol{u}^{(0)}$ does not depend on $\boldsymbol{y}$.

Equations (18), (20) and (30) constitute a system of equations for $p^{(0)}$. Multiplying (18) by $\bar{p}^{(0)}$, integrating the result over $Y$ and applying (20), (30) together with the $Y$-periodic condition on $p^{(0)}$, we get

$$
\int_{Y} K_{\alpha \beta} \partial_{y_{\alpha}} p^{(0)} \overline{\partial_{y_{\beta}} p^{(0)}} d \boldsymbol{y}=0
$$

This implies

$$
\partial_{y_{\beta}} p^{(0)}=0
$$

i.e. $p^{(0)}$ does not depend on $\boldsymbol{y}$.

Next, we consider the $\mathcal{O}\left(\epsilon^{-1}\right)$ equations. By using (35) and (36), (22) and (24) reduce to

$$
(P 1)\left\{\begin{array}{l}
K_{\alpha \beta} \partial_{y_{\alpha}} \partial_{y_{\beta}} p^{(1)}=0, x_{3} \neq h\left(y_{1}, y_{2}\right), \\
{\left[\left(K_{\alpha \beta} \partial_{y_{\beta}} p^{(1)}+K_{\alpha j}\left(\partial_{x_{j}} p^{(0)}-\rho_{l} \omega^{2} u_{j}^{(0)}\right)\right)\left(\partial_{y_{\alpha}} h\right)\right]=0 .}
\end{array}\right.
$$

To solve $(\mathrm{P} 1)$, we introduce a new variable $\phi_{j}\left(x_{3}, \boldsymbol{y}\right)$ by writing $p^{(1)}$ in the form

$$
p^{(1)}(\boldsymbol{x}, \boldsymbol{y}, t)=\phi_{j}\left(x_{3}, \boldsymbol{y}\right)\left(\partial_{x_{j}} p^{(0)}(\boldsymbol{x}, t)-\rho_{l} \omega^{2} u_{j}^{(0)}(\boldsymbol{x}, t)\right) .
$$

Substituting (38) into (P1), we can see that $p^{(1)}(\boldsymbol{x}, \boldsymbol{y}, t)$ in (38) will solve (P1) if $\phi_{j}\left(x_{3}, \boldsymbol{y}\right)$ satisfies

$$
(A P 1)\left\{\begin{array}{l}
K_{\alpha \beta} \partial_{y_{\alpha}} \partial_{y_{\beta}} \phi_{j}=0, x_{3} \neq h\left(y_{1}, y_{2}\right) \\
{\left[\left(K_{\alpha \beta} \partial_{y_{\beta}} \phi_{j}+K_{\alpha j}\right)\left(\partial_{y_{\alpha}} h\right)\right]=0}
\end{array}\right.
$$

We also require $\phi_{j}\left(x_{3}, \boldsymbol{y}\right)$ to be $Y$-periodic in the $\boldsymbol{y}$ variable, continuous in $Y$ and to have zero average over $Y$. These conditions uniquely determine $\phi_{j}$. 

$\boldsymbol{u}^{(1)}$ :

Similarly, by applying (35) and (36) to equations (21) and (23), we get the following system of equations for

$$
(P 2):\left\{\begin{array}{l}
\partial_{y_{\beta}}\left(C_{i \beta k \delta} \partial_{y_{\delta}} u_{k}^{(1)}\right)=\mathbf{0}, x_{3} \neq h\left(y_{1}, y_{2}\right), \\
{\left[\left(C_{i \beta k \delta} \partial_{y_{\delta}} u_{k}^{(1)}+C_{i \beta k l} \partial_{x_{l}} u_{k}^{(0)}-\tilde{\alpha} p^{(0)} \delta_{i \beta}\right)\left(\partial_{y_{\beta}} h\right)\right]=\mathbf{0} .}
\end{array}\right.
$$

To solve (P2), we introduce a new variable $\chi_{k m n}$ by writing $u_{k}^{(1)}$ in the form

$$
u_{k}^{(1)}(\boldsymbol{x}, \boldsymbol{y}, t)=\chi_{k m n}\left(x_{3}, \boldsymbol{y}\right)\left(\partial_{x_{n}} u_{m}^{(0)}(\boldsymbol{x}, t)+A_{m n} p^{(0)}(\boldsymbol{x}, t)\right),
$$

where $A_{m n}$ is an absolute constant matrix such that

$$
\left\{\begin{array}{l}
C_{i \beta m n}^{+} A_{m n}=-\tilde{\alpha}^{+} \delta_{i \beta} \\
C_{i \beta m n}^{-} A_{m n}=-\tilde{\alpha}^{-} \delta_{i \beta}
\end{array}\right.
$$

By direct calculation, it can be seen that

$$
\begin{aligned}
& A_{11}=A_{22}=\frac{\tilde{\alpha}^{-}\left(\lambda^{+}+\mu^{+}\right)-\tilde{\alpha}^{+}\left(\lambda^{-}+\mu^{-}\right)}{\left(\lambda^{-}+\mu^{-}\right)\left(2 \lambda^{+}+3 \mu^{+}\right)-\left(\lambda^{+}+\mu^{+}\right)\left(2 \lambda^{-}+3 \mu^{-}\right)}, \\
& A_{33}=\frac{\tilde{\alpha}^{-}\left(2 \lambda^{+}+3 \mu^{+}\right)-\tilde{\alpha}^{+}\left(2 \lambda^{-}+3 \mu^{-}\right)}{\left(\lambda^{-}+\mu^{-}\right)\left(2 \lambda^{+}+3 \mu^{+}\right)-\left(\lambda^{+}+\mu^{+}\right)\left(2 \lambda^{-}+3 \mu^{-}\right)}, \\
& A_{m n}=0 \text { if } m \neq n,
\end{aligned}
$$

where $\lambda^{+}, \mu^{+}, \lambda^{-}$and $\mu^{-}$are the Lamé coefficients of the material in $Y^{+}$and $Y^{-}$, respectively.

Substituting (40) into (P2) gives

$$
\left\{\begin{array}{l}
C_{i \beta k \delta}\left(\partial_{y_{\beta}} \partial_{y_{\delta}} \chi_{k m n}\right)\left(\partial_{x_{n}} u_{m}^{(0)}+A_{m n} p^{(0)}\right)=0, x_{3} \neq h\left(y_{1}, y_{2}\right), \\
{\left[\left(C_{i \beta k \delta} \partial_{y_{\delta}} \chi_{k m n}+C_{i \beta m n}\right)\left(\partial_{y_{\beta}} h\right)\right]\left(\partial_{x_{n}} u_{m}^{(0)}+A_{m n} p^{(0)}\right)=0 .}
\end{array}\right.
$$

Therefore, (40) solves (P2) if $\chi_{k m n}$ satisfies

$$
(A P 2)\left\{\begin{array}{l}
C_{i \beta k \delta}\left(\partial_{y_{\beta}} \partial_{y_{\delta}} \chi_{k m n}\right)=\mathbf{0}, x_{3} \neq h\left(y_{1}, y_{2}\right) \\
{\left[\left(C_{i \beta k \delta} \partial_{y_{\delta}} \chi_{k m n}+C_{i \beta m n}\right)\left(\partial_{y_{\beta}} h\right)\right]=\mathbf{0} .}
\end{array}\right.
$$

We also require $\chi_{k m n}$ to be continuous in $Y, Y$-periodic in the $y$ variable and to have zero average over $Y$. These conditions uniquely determine $\chi_{k m n}$.

Next, we consider the $\mathcal{O}\left(\epsilon^{0}\right)$ equations. We integrate (25) with respect to $y$ over $Y$ and divide it by the area of $Y$, which is denoted by $A$. The first and the third term can be converted to integrals around $\Gamma$ and $\partial Y$ by the divergence theorem. The integrals along $\partial Y$ vanish because of the assumed $Y$-periodicity of $\boldsymbol{u}^{(1)}$ and $\boldsymbol{u}^{(2)}$. We thus obtain

$$
\begin{aligned}
& -A^{-1} \int_{\Gamma}\left[\left(C_{i \beta k \delta} \partial_{y_{\delta}} u_{k}^{(2)}+C_{i \beta k l} \partial_{x_{l}} u_{k}^{(1)}-\tilde{\alpha} \delta_{i \beta} p^{(1)}\right)\left(\partial_{y_{\beta}} h\right)\right] \frac{d \sigma}{\left|\nabla_{\boldsymbol{y}} h\right|} \\
+ & A^{-1} \int_{Y} \partial_{x_{j}}\left(C_{i j k \delta} \partial_{y_{\delta}} u_{k}^{(1)}\right) d \boldsymbol{y}+A^{-1} \int_{Y} \breve{\alpha}_{i \gamma} \partial_{y_{\gamma}} p^{(1)} d \boldsymbol{y} \\
+ & A^{-1} \int_{Y} \partial_{x_{j}}\left(C_{i j k l} \partial_{x_{l}} u_{k}^{(0)}\right) d \boldsymbol{y}+\omega^{2}<\breve{\rho}_{i j}>u_{j}^{(0)} \\
+ & <\breve{\alpha}_{i j}>\partial_{x_{j}} p^{(0)}-<\tilde{\alpha}>\partial_{x_{i}} p^{(0)}=0,
\end{aligned}
$$


where $<\cdot>$ is the "averaging operator" defined as

$$
<\cdot>:=A^{-1} \int_{Y} \cdot d \boldsymbol{y}
$$

By using (27), the first integral in (42) can be written as

$$
\begin{aligned}
& -A^{-1} \int_{\Gamma}\left[C_{i 3 k \delta} \partial_{y_{\delta}} u_{k}^{(1)}+C_{i 3 k l} \partial_{x_{l}} u_{k}^{(0)}-\tilde{\alpha} \delta_{i \beta} p^{(0)}\right] \frac{d \sigma}{\left|\nabla_{\boldsymbol{y}} h\right|} \\
= & -A^{-1} \int_{\Gamma}\left[C_{i 3 k \delta} \partial_{y_{\delta}} u_{k}^{(1)}\right] \frac{d \sigma}{\left|\nabla_{\boldsymbol{y}} h\right|}-A^{-1}\left(\left[C_{i 3 k l}\right] \partial_{x_{l}} u_{k}^{(0)}-[\tilde{\alpha}] \delta_{i 3} p^{(0)}\right) \int_{\Gamma} \frac{d \sigma}{\left|\nabla_{\boldsymbol{y}} h\right|} \\
= & -A^{-1} \int_{\Gamma}\left[C_{i 3 k \delta} \partial_{y_{\delta}} u_{k}^{(1)}\right] \frac{d \sigma}{\left|\nabla_{\boldsymbol{y}} h\right|}-A^{-1}\left(\left[C_{i 3 k l}\right] \partial_{x_{l}} u_{k}^{(0)}-[\tilde{\alpha}] \delta_{i 3} p^{(0)}\right) \partial_{x_{3}} A_{1},
\end{aligned}
$$

where $A_{1}$ is the area of $Y^{+}$. The last equality in (43) follows from the coarea formula [1], which states that

$$
\int_{\Gamma} \frac{d \sigma}{\left|\nabla_{\boldsymbol{y}} h\right|}=\partial_{x_{3}} A_{1}
$$

By direct calculation and using the facts that $Y^{+}$and $Y^{-}$are functions of $x_{3}$ only, and $\boldsymbol{u}^{(0)}, A_{m n}$ and $p^{(0)}$ do not depend on $\boldsymbol{y}$, the second integral in (42) can be converted to

$$
<C_{i j k \delta} \partial_{y_{\delta}} \chi_{k m n}>\partial_{x_{j}}\left(\partial_{x_{n}} u_{m}^{(0)}+A_{m n} p^{(0)}\right)+<C_{i 3 k \delta} \partial_{x_{3}} \partial_{y_{\delta}} \chi_{k m n}>\left(\partial_{x_{n}} u_{m}^{(0)}+A_{m n} p^{(0)}\right) .
$$

The fourth integral in (42) is equal to $\partial_{x_{j}} \partial_{x_{l}} u_{k}^{(0)}<C_{i j k l}>$ because $\boldsymbol{u}^{(0)}$ is not a function of $\boldsymbol{y}$ and $C_{i j k l}$ are constants in $Y^{+}$and $Y^{-}$. Realizing that $\left\langle C_{i j k l}>\right.$ depends only on $x_{3}$ and

$$
<C_{i j k l}>=A^{-1}\left(A_{1} C_{i j k l}^{+}+\left(A-A_{1}\right) C_{i j k l}^{-}\right),
$$

we may further write $\left(\partial_{x_{j}} \partial_{x_{l}} u_{k}^{(0)}\right)<C_{i j k l}>$ as

$$
\begin{aligned}
& \left(\partial_{x_{j}} \partial_{x_{l}} u_{k}^{(0)}\right)<C_{i j k l}> \\
= & \partial_{x_{j}}\left(\partial_{x_{l}} u_{k}^{(0)}<C_{i j k l}>\right)+A^{-1}\left(\partial_{x_{3}} A_{1}\right)\left[C_{i 3 k l}\right] \partial_{x_{l}} u_{k}^{(0)} .
\end{aligned}
$$

Similarly, the last term in (42) can be written as

$$
<\tilde{\alpha}>\partial_{x_{i}} p^{(0)}=\partial_{x_{i}}\left(<\tilde{\alpha}>p^{(0)}\right)+A^{-1}\left(\partial_{x_{i}} A_{1}\right) \delta_{i 3}[\tilde{\alpha}] .
$$

Finally, we substitute (43), (44), (45), (46), (38) and (40) into (42). Note that the last term in (43) cancels with the last term in (45) and (46). This yields a new equation

$$
\begin{aligned}
& -\left(A^{-1} \int_{\Gamma}\left[C_{i 3 k \delta} \partial_{y_{\delta}} \chi_{k m n}\right] \frac{d \sigma}{\left|\nabla_{\boldsymbol{y}} h\right|}\right)\left(\partial_{x_{n}} u_{m}^{(0)}+A_{m n} p^{(0)}\right) \\
& +<C_{i j k \delta} \partial_{y_{\delta}} \chi_{k m n}>\partial_{x_{j}}\left(\partial_{x_{n}} u_{m}^{(0)}+A_{m n} p^{(0)}\right) \\
& +<C_{i 3 k \delta} \partial_{x_{3}} \partial_{y_{\delta}} \chi_{k m n}>\left(\partial_{x_{n}} u_{m}^{(0)}+A_{m n} p^{(0)}\right)+\left(\partial_{x_{j}} p^{(0)}-\rho_{l} \omega^{2} u_{j}^{(0)}\right)<\breve{\alpha}_{i \gamma} \partial_{y_{\gamma}} \phi_{j}> \\
& +\partial_{x_{j}}\left(\partial_{x_{l}} u_{k}^{(0)}<C_{i j k l}>\right)+\partial_{x_{i}}\left(<\tilde{\alpha}>p^{(0)}\right)+\omega^{2}<\breve{\rho}_{i j}>u_{j}^{(0)}+<\breve{\alpha}_{i j}>\partial_{x_{j}} p^{(0)}=0 .
\end{aligned}
$$


Introduce the "effective" parameters

$$
\begin{aligned}
C_{i j m n}^{e f f}: & =<C_{i j k \delta} \partial_{y_{\delta}} \chi_{k m n}>+<C_{i j n m}>, \\
M_{i m n}^{e f f}: & =-A^{-1} \int_{\Gamma}\left[C_{i 3 k \delta} \partial_{y_{\delta}} \chi_{k m n}\right] \frac{d \sigma}{\left|\nabla_{\boldsymbol{y}} h\right|} \\
& -\partial_{x_{3}}<C_{i j k \delta} \partial_{y_{\delta}} \chi_{k m n}>+<C_{i 3 k \delta} \partial_{x_{3}} \partial_{y_{\delta}} \chi_{k m n}> \\
\breve{\alpha}_{i j}^{e f f}: & =<\breve{\alpha}_{i \gamma} \partial_{y_{\gamma}} \phi_{j}+\breve{\alpha}_{i j}>+A_{m n}<C_{i j k \delta} \partial_{y_{\delta}} \chi_{k m n}>, \\
\breve{\rho}_{i j}^{e f f}: & =<\breve{\rho}_{i j}>-\rho_{l}<\breve{\alpha}_{i \gamma} \partial_{y_{\gamma}} \phi_{j}>.
\end{aligned}
$$

Then (47) can be rewritten as

$$
\partial_{x_{j}}\left(C_{i j m n}^{e f f} \partial_{x_{n}} u_{m}^{(0)}\right)+\omega^{2} \breve{\rho}_{i j}^{e f f} u_{j}^{(0)}-M_{i m n}^{e f f} \partial_{x_{n}} u_{m}^{(0)}=-\breve{\alpha}_{i j}^{e f f} \partial_{x_{j}} p^{(0)},
$$

which is the homogenized equation of (5).

Similarly, we first apply $<\cdot>$ to (26). Using the divergence theorem on the first and fifth term, we convert the integrals to be boundary integrals of jumps around $\Gamma$. Secondly, we apply equation (28) to rewrite the boundary integral of the jump. Thirdly, we replace $u_{k}^{(1)}$ and $p^{(1)}$ by using (38) and (40). Finally, (26) becomes

$$
\begin{aligned}
& \left(\frac{\mathcal{I}}{\omega}\right)\left(A^{-1} \int_{\Gamma}\left[K_{3 \beta} \partial_{y_{\beta}} \phi_{j}+K_{3 j}\right] \frac{d \sigma}{\left|\nabla_{\boldsymbol{y}} h\right|}\right)\left(\partial_{x_{j}} p^{(0)}-\rho_{l} \omega^{2} u_{j}^{(0)}\right) \\
- & \left(\frac{\mathcal{I}}{\omega}\right)<K_{i j}>\partial_{x_{i}} \partial_{x_{j}} p^{(0)}-<\tilde{\alpha} \partial_{y_{\beta}} \chi_{\beta m n}>\left(\partial_{x_{n}} u_{m}^{(0)}+A_{m n} p^{(0)}\right) \\
- & \left(\frac{\mathcal{I}}{\omega}\right)\left(\partial_{x_{j}} p^{(0)}-\rho_{l} \omega^{2} u_{j}^{(0)}\right)<K_{3 \beta} \partial_{x_{3}}\left(\partial_{y_{\beta}} \phi_{j}\right)> \\
- & \left(\frac{\mathcal{I}}{\omega}\right)\left(\partial_{x_{i}} \partial_{x_{j}} p^{(0)}-\rho_{l} \omega^{2} \partial_{x_{i}} u_{j}^{(0)}\right)<K_{i \beta} \partial_{y_{\beta}} \phi_{j}> \\
- & <\tilde{\alpha}>\partial_{x_{i}} u_{i}^{(0)}-<\tilde{\beta}>p^{(0)}+\mathcal{I} \omega \rho_{l}<K_{i j}>\partial_{x_{i}} u_{j}^{(0)}=0 .
\end{aligned}
$$

Introducing another set of "effective" parameters $K_{i j}^{e f f}, L_{n m}^{e f f}, Q_{j}^{e f f}$ and $\tilde{\beta}^{\text {eff }}$,

$$
\begin{aligned}
& K_{i j}^{e f f}:=<K_{i j}>+<K_{i \beta} \partial_{y_{\beta}} \phi_{j}>, \\
& L_{n m}^{e f f}:=<\tilde{\alpha} \partial_{y_{\beta}} \chi_{\beta m n}>+<\tilde{\alpha}>\delta_{m n}, \\
& Q_{j}^{e f f}:=-A^{-1} \int_{\Gamma}\left[K_{3 \beta} \partial_{y_{\beta}} \phi_{j}+K_{3 j}\right] \frac{d \sigma}{\left|\nabla_{\boldsymbol{y}} h\right|}+<K_{3 \beta} \partial_{x_{3}}\left(\partial_{y_{\beta}} \phi_{j}\right)>, \\
& \tilde{\beta}^{e f f}:=<\tilde{\beta}>+A_{m n}<\tilde{\alpha} \partial_{y_{\beta}} \chi_{\beta m n}>,
\end{aligned}
$$

(53) can be written as

$$
\begin{aligned}
& \left(-\frac{\mathcal{I}}{\omega}\right) K_{i j}^{e f f}\left(\partial_{x_{i}} \partial_{x_{j}} p^{(0)}-\rho_{l} \omega^{2} \partial_{x_{i}} u_{j}^{(0)}\right)-L_{n m}^{e f f} \partial_{x_{n}} u_{m}^{(0)} \\
= & \tilde{\beta}^{e f f} p^{(0)}+\frac{\mathcal{I}}{\omega} Q_{j}^{e f f}\left(\partial_{x_{j}} p^{(0)}-\rho_{l} \omega^{2} u_{j}^{(0)}\right) .
\end{aligned}
$$

This is the homogenized equation of (6).

We summarize our discussion as follows. 
Theorem 3.1. Let $\boldsymbol{u}(\boldsymbol{x}, t, \epsilon)$ and $p(\boldsymbol{x}, t, \epsilon)$ satisfy the dynamic equation (5) and the continuity equation (6) on both sides of the periodic surface $x_{3}=h\left(x_{1}, x_{2}\right)$ with constant solid density $\rho_{s}$, fluid density $\rho_{l}$, Darcy permeability $\boldsymbol{K}$, porosity $f$, effective parameters $\tilde{\alpha}$ and $\tilde{\beta}$ on each side. Suppose $\boldsymbol{u}(\boldsymbol{x}, t, \epsilon)$ and $p(\boldsymbol{x}, t, \epsilon)$ also satisfy the continuity conditions (7)-(10) across the interface $x_{3}=h\left(x_{1}, x_{2}\right)$.

If $\boldsymbol{u}(\boldsymbol{x}, t, \epsilon)$ and $p(\boldsymbol{x}, t, \epsilon)$ have the asymptotic forms (15) and (16), respectively. Then $\boldsymbol{u}^{(0)}(\boldsymbol{x}, t)$ and $p^{(0)}(\boldsymbol{x}, t)$ satisfy the following system of homogenized equations in $0<x_{3}<a$,

$$
\begin{aligned}
& \partial_{x_{j}}\left(C_{i j m n}^{e f f} \partial_{x_{n}} u_{m}^{(0)}\right)+\omega^{2} \breve{\rho}_{i j}^{e f f} u_{j}^{(0)}-M_{i m n}^{e f f} \partial_{x_{n}} u_{m}^{(0)}=-\breve{\alpha}_{i j}^{e f f} \partial_{x_{j}} p^{(0)}, \\
& \left(-\frac{\mathcal{I}}{\omega}\right) K_{i j}^{e f f}\left(\partial_{x_{i}} \partial_{x_{j}} p^{(0)}-\rho_{l} \omega^{2} \partial_{x_{i}} u_{j}^{(0)}\right)-L_{n m}^{e f f} \partial_{x_{n}} u_{m}^{(0)}=\tilde{\beta}^{e f f} p^{(0)} \\
& +\frac{\mathcal{I}}{\omega} Q_{j}^{e f f}\left(\partial_{x_{j}} p^{(0)}-\rho_{l} \omega^{2} u_{j}^{(0)}\right) .
\end{aligned}
$$

Here $a$ is as defined in Figure $1,<\cdot>$ the average operator, and the effective parameters are defined in (48) to (51) and (54) to (57).

\section{A Numerical Example}

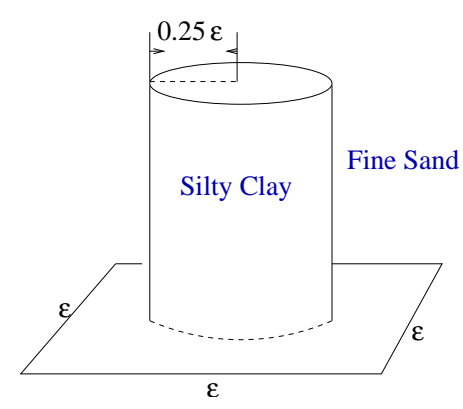

Figure 3: Single cell of the periodic interface

In this section, we will construct the effective parameters of a special case of the previous discussion. For simplicity, the interface is assumed to consist of very narrow truncated uniform cylinders with radius equal $0.25 \epsilon$ ( See Figure 3). In this case, $h=x_{1}^{2}+x_{2}^{2}=(0.25 \epsilon)^{2}, \epsilon=0.1$, and the unit normal vectors on the interface are proportional to $\left(\epsilon^{-1} \partial_{y_{1}} h, \epsilon^{-1} \partial_{y_{2}} h, 0\right)$ rather than $\left(\epsilon^{-1} \partial_{y_{1}} h, \epsilon^{-1} \partial_{y_{2}} h,-1\right)$. Accordingly, the effective parameter $M_{i n m}^{e f f}$ and $Q_{j}^{e f f}$ are modified to be

$$
\begin{aligned}
M_{i m n}^{e f f}: & =-\partial_{x_{3}}<C_{i j k \delta} \partial_{y_{\delta}} \chi_{k m n}>+<C_{i 3 k \delta} \partial_{x_{3}} \partial_{y_{\delta}} \chi_{k m n}>, \\
Q_{j}^{e f f}: & =<K_{3 \beta} \partial_{x_{3}}\left(\partial_{y_{\beta}} \phi_{j}\right)>,
\end{aligned}
$$

whereas the other effective parameters remain unchanged.

Suppose the upper layer is of fine sand and the lower layer is of silty clay. Using the experimental data in [13] and the formulas (58)-(71) in [14], we list the Lamé coefficients and Darcy tensor of each layer in Table 1. The frequency $\omega$ is chosen to be $200 \mathrm{~Hz}$.

The effective parameters are computed using the solutions of the two auxiliary problems $(A P 1)$ and $(A P 2)$. Part of the solution of these problems is given in Figure 4 - Figure 6. The effective parameters are listed below. 


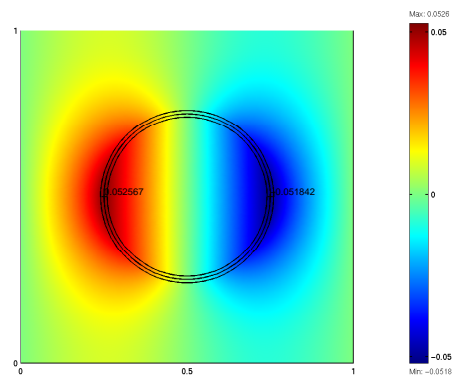

(a) Real part

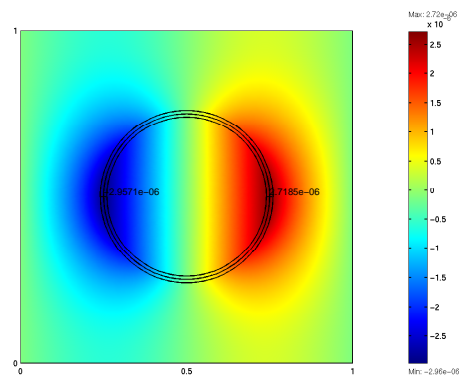

(b) Imaginary part

Figure 4: Solution to $(A P 1): \phi_{1}$

$$
\begin{aligned}
& \left(\begin{array}{lll}
C_{1111}^{\text {eff }} & C_{1112}^{\text {eff }} & C_{1113}^{\text {eff }} \\
C_{1121}^{\text {eff }} & C_{1122}^{\text {eff }} & C_{1123}^{\text {eff }} \\
C_{1131}^{\text {eff }} & C_{1132}^{\text {eff }} & C_{1133}^{\text {eff }}
\end{array}\right)=10^{9} \times \\
& \left(\begin{array}{lll}
1.19242-0.00047 \mathcal{I} & 0.00000-0.00000 \mathcal{I} & 0.00000+0.00000 \mathcal{I} \\
0.00000-0.00000 \mathcal{I} & 1.18068-0.00009 \mathcal{I} & 0.00000+0.00000 \mathcal{I} \\
0.00000+0.00000 \mathcal{I} & 0.00000+0.00000 \mathcal{I} & 1.41625-0.00010 \mathcal{I}
\end{array}\right) ; \\
& \left(\begin{array}{llll}
C_{1211}^{\text {eff }} & C_{1212}^{\text {eff }} & C_{1213}^{\text {eff }} \\
C_{1221}^{\text {fff }} & C_{1222}^{\text {eff }} & C_{1223}^{\text {eff }} \\
C_{1231}^{\text {eff }} & C_{123 f}^{\text {eff }} & C_{1233}^{\text {eff }}
\end{array}\right)=\left(\begin{array}{lll}
C_{2111}^{\text {eff }} & C_{2112}^{\text {eff }} & C_{2113}^{\text {eff }} \\
C_{2121}^{\text {eff }} & C_{212}^{\text {eff }} & C_{2123}^{\text {eff }} \\
C_{2131}^{\text {eff }} & C_{2132}^{\text {eff }} & C_{2133}^{\text {eff }}
\end{array}\right)=10^{6} \times \\
& \left(\begin{array}{llll}
0.00000-0.00000 \mathcal{I} & 5.87099-0.18940 \mathcal{I} & 0.00000+0.00000 \mathcal{I} \\
5.87099-0.18940 \mathcal{I} & 0.00000-0.00000 \mathcal{I} & 0.00000+0.00000 \mathcal{I} \\
0.00000+0.00000 \mathcal{I} & 0.00000+0.00000 \mathcal{I} & 0.00000+0.00000 \mathcal{I}
\end{array}\right) ;
\end{aligned}
$$

Table 1: Physical parameters of the poroelastic media

\begin{tabular}{|c||c|c|}
\hline \hline & fine sand & silty clay \\
\hline$\mu$ & $7.12 \times 10^{6}-2.3 \times 10^{5} \mathcal{I}$ & $7.86 \times 10^{6}-2.5 \times 10^{4} \mathcal{I}$ \\
\hline$\lambda$ & $1.68 \times 10^{9}-1.04263 \times 10^{5} \mathcal{I}$ & $3.5 \times 10^{8}-9.6 \times 10^{4} \mathcal{I}$ \\
\hline$K_{i j}$ & $\left(3.09 \times 10^{-11}-5.55 \times 10^{-16} \mathcal{I}\right) \delta_{i j}$ & $\left(5.15 \times 10^{-11}-2.34 \times 10^{-15} \mathcal{I}\right) \delta_{i j}$ \\
\hline
\end{tabular}



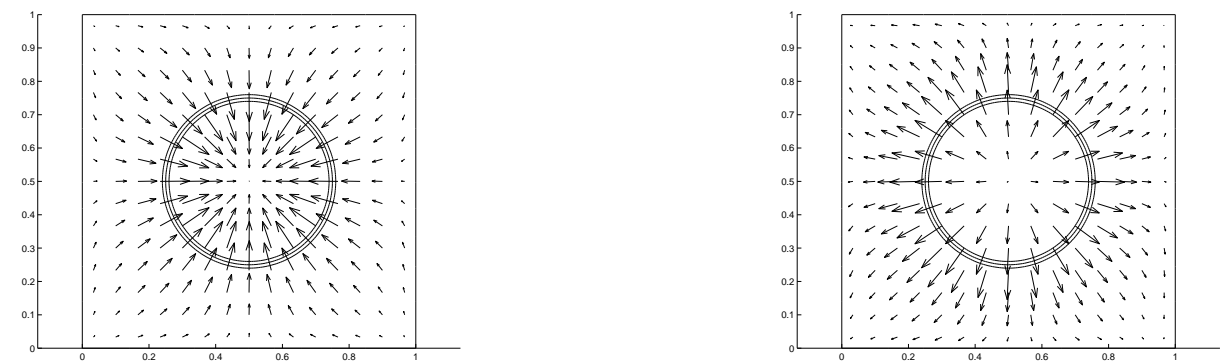

(a) Real part

(b) Imaginary part

Figure 5: Solution to $(A P 1)$ : Vector plot of $\left(\phi_{1}, \phi_{2}\right)$

$$
\begin{aligned}
& \left(\begin{array}{lll}
C_{1311}^{e f f} & C_{1312}^{e f f} & C_{1313}^{e f f} \\
C_{1321}^{e f f} & C_{132}^{e f f} & C_{1323}^{e f f} \\
C_{1331}^{e f f} & C_{1332}^{e f f} & C_{1333}^{e f f}
\end{array}\right)=\left(\begin{array}{ccc}
C_{311}^{e f f} & C_{3112}^{\text {eff }} & C_{3113}^{\text {eff }} \\
C_{3121}^{\text {eff }} & C_{3122}^{\text {eff }} & C_{3123}^{\text {eff }} \\
C_{3131}^{\text {eff }} & C_{3132}^{\text {eff }} & C_{3133}^{\text {eff }}
\end{array}\right)=10^{6} \times \\
& \left(\begin{array}{ccc}
0.00000+0.00000 \mathcal{I} & 0.00000+0.00000 \mathcal{I} & 5.18393-0.16727 \mathcal{I} \\
0.00000+0.00000 \mathcal{I} & 0.00000+0.00000 \mathcal{I} & -0.00000+0.00000 \mathcal{I} \\
5.18393-0.16727 \mathcal{I} & -0.00000+0.00000 \mathcal{I} & 0.00000+0.00000 \mathcal{I}
\end{array}\right)
\end{aligned}
$$

$\left(\begin{array}{ccc}C_{2211}^{e f f} & C_{2212}^{\text {eff }} & C_{2213}^{\text {eff }} \\ C_{2221}^{\text {eff }} & C_{222 \mathrm{f} f}^{\text {eff }} & C_{2223}^{\text {eff }} \\ C_{2231}^{\text {eff }} & C_{2232}^{\text {eff }} & C_{2233}^{\text {eff }}\end{array}\right)=10^{9} \times$

$\left(\begin{array}{lll}1.18068-0.00009 \mathcal{I} & 0.00000-0.00000 \mathcal{I} & 0.00000+0.00000 \mathcal{I} \\ 0.00000-0.00000 \mathcal{I} & 1.19242-0.00047 \mathcal{I} & 0.00000+0.00000 \mathcal{I} \\ 0.00000+0.00000 \mathcal{I} & 0.00000+0.00000 \mathcal{I} & 1.41625-0.00010 \mathcal{I}\end{array}\right)$ 


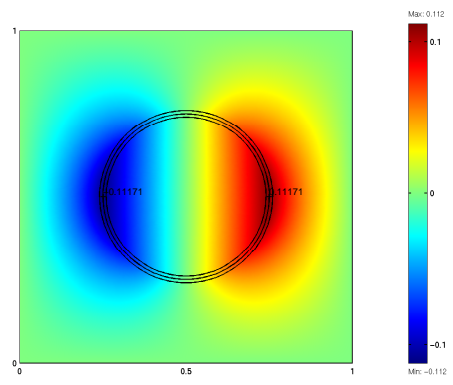

(a) Real part

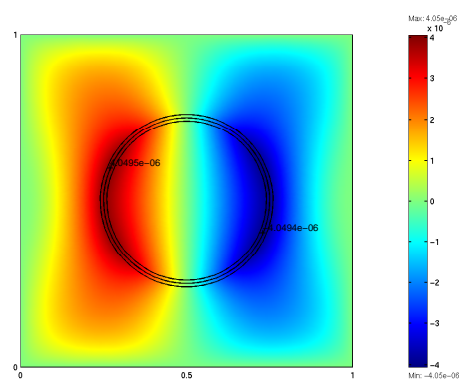

(b) Imaginary part

Figure 6: Solution to $(A P 2): \chi_{111}$

$$
\begin{aligned}
& \left(\begin{array}{ccc}
C_{2311}^{e f f} & C_{2312}^{e f f} & C_{2313}^{e f f} \\
C_{2321}^{e f f} & C_{2322}^{e f f} & C_{2323}^{e f f} \\
C_{2331}^{e f f} & C_{2332}^{e f f} & C_{2333}^{e f f}
\end{array}\right)=\left(\begin{array}{ccc}
C_{3211}^{e f f} & C_{3212}^{e f f} & C_{3213}^{e f f} \\
C_{3221}^{e f f} & C_{3222}^{e f f} & C_{3223}^{e f f} \\
C_{3231}^{e f f} & C_{3232}^{e f f} & C_{3233}^{e f f}
\end{array}\right)=10^{6} \times \\
& \left(\begin{array}{cccc}
0.00000+0.00000 \mathcal{I} & 0.00000+0.00000 \mathcal{I} & -0.00000+0.00000 \mathcal{I} \\
0.00000+0.00000 \mathcal{I} & 0.00000+0.00000 \mathcal{I} & 5.18393-0.16727 \mathcal{I} \\
-0.00000+0.00000 \mathcal{I} & 5.18393-0.16727 \mathcal{I} & 0.00000+0.00000 \mathcal{I}
\end{array}\right)
\end{aligned}
$$

$$
\begin{aligned}
& \left(\begin{array}{lll}
C_{3311}^{e f f} & C_{3312}^{\text {eff }} & C_{3313}^{\text {eff }} \\
C_{3321}^{\text {eff }} & C_{3322}^{\text {eff }} & C_{3323}^{\text {eff }} \\
C_{3331}^{\text {eff }} & C_{3332}^{\text {eff }} & C_{3333}^{\text {eff }}
\end{array}\right)=10^{9} \times \\
& \left(\begin{array}{lll}
1.18180-0.00013 \mathcal{I} & 0.00000-0.00000 \mathcal{I} & 0.00000+0.00000 \mathcal{I} \\
0.00000-0.00000 \mathcal{I} & 1.18180-0.00013 \mathcal{I} & 0.00000+0.00000 \mathcal{I} \\
0.00000+0.00000 \mathcal{I} & 0.00000+0.00000 \mathcal{I} & 1.42800-0.00048 \mathcal{I}
\end{array}\right)
\end{aligned}
$$




$$
\begin{aligned}
& M_{i m n}^{e f f}=0, \forall i, m, n \\
& \breve{\alpha}_{i j}^{e f f}=10^{3} \cdot\left(\begin{array}{ccc}
-1.77942-0.05891 \mathcal{I} & 0.00000+0.00000 \mathcal{I} & 0.00000+0.00000 \mathcal{I} \\
0.00000+0.00000 \mathcal{I} & -1.77942-0.05891 \mathcal{I} & 0.00000+0.00000 \mathcal{I} \\
0.00000+0.00000 \mathcal{I} & 0.00000+0.00000 \mathcal{I} & -1.77170-0.05891 \mathcal{I}
\end{array}\right) ; \\
& \breve{\rho}_{i j}^{\text {eff }}=10^{3} \cdot\left(\begin{array}{ccc}
1.87055-0.00001 \mathcal{I} & 0.00000+0.00000 \mathcal{I} & 0.00000+0.00000 \mathcal{I} \\
0.00000+0.00000 \mathcal{I} & 1.87055-0.00001 \mathcal{I} & 0.00000+0.00000 \mathcal{I} \\
0.00000+0.00000 \mathcal{I} & 0.00000+0.00000 \mathcal{I} & 1.87055-0.00001 \mathcal{I}
\end{array}\right) ; \\
& K_{i j}^{e f f}=10^{-11} \cdot\left(\begin{array}{ccc}
3.40805-0.00009 \mathcal{I} & -0.00000-0.00000 \mathcal{I} & 0.00000+0.00000 \mathcal{I} \\
-0.00000-0.00000 \mathcal{I} & 3.40805-0.00009 \mathcal{I} & 0.00000+0.00000 \mathcal{I} \\
0.00000+0.00000 \mathcal{I} & 0.00000+0.00000 \mathcal{I} & 3.49347-0.00009 \mathcal{I}
\end{array}\right) ; \\
& L_{n m}^{e f f}=\left(\begin{array}{ccc}
1.90292+0.00003 \mathcal{I} & -0.00000+0.00000 \mathcal{I} & 0.00000+0.00000 \mathcal{I} \\
-0.00000+0.00000 \mathcal{I} & 1.90292+0.00003 \mathcal{I} & 0.00000+0.00000 \mathcal{I} \\
0.00000+0.00000 \mathcal{I} & 0.00000+0.00000 \mathcal{I} & 1.59361+0.00000 \mathcal{I}
\end{array}\right) \\
& Q_{j}^{e f f}=\left(\begin{array}{l}
0 \\
0 \\
0
\end{array}\right) \\
& \tilde{\beta}^{e f f}=-5.7549 \times 10^{-7}-1.9171 \times 10^{-8} \mathcal{I} \text {. }
\end{aligned}
$$

Acknowledgement This work was supported in part by grant INT-9726213.

\section{References}

[1] C. Bandle. Isoperimetric Inequalities and Applications, Pitman, 1980

[2] Z. Lin. Some Direct and Inverse Problems Involving Inhomogeneous Media, Ph.D. Thesis, University of Delaware, 1998

[3] W. Kohler and G.C. Papanicolaou and S. Varadhan. Boundary and interface problems in regions with very rough boundaries in Multiple Scattering and Waves in Random Media, P. Chow and W. Kohler and G.C. Papanicolaou, eds., pp. 165-197, North-Holland, Amsterdam, 1981

[4] J. Nevard and J.B. Keller. Homogenization of Rough Boundaries and Interfaces, SIAM J. Appl. Math., Vol. 57, No. 6, pp. 1660-1686, 1997

[5] M.A. Biot. Theory of propagation of elastic waves in a fluid-saturated porous solid. J. Acoustical Society of America, Vol. 28, No. 1, pp. 168-178, 1956

[6] M.A. Biot. Generalized theory of acoustic propagation in porous dissipative media. J. Acoustical Society of America, Vol. 34, pp. 1254-1264, 1962.

[7] M.A. Biot. Mechanics of deformation and acoustic propagation in porous media.Journal of Applied Physics. Vol. 33, pp. 1482-1498, 1962

[8] R. Burridge and J.B. Keller. Poroelasticity equations derived from microstructure. J. Acoustical Society of America, Vol. 70, No. 4, pp. 1140-1146, 1981

[9] R.P. Gilbert and A. Mikelić. Homogenizing the acoustic properties of the seabed: Part I. Nonlinear Analysis, Theory, Methods and applications, Vol. 40, pp. 185-212, 2000 
[10] J.L. Auriault. Dynamic behavior of a porous medium saturated by a Newtonian fluid. Int. J. Engng. Sci. Vol. 18, pp. 775-785, 1980

[11] J.L. Auriault and L. Borne and R. Chambon. Dynamics of porous saturated media, checking of the generalized law of Darcy. J. Acoustical Society of America, Vol. 77, No. 5, pp. 1641-1650, 1985

[12] C. Boutin and G. Bonnet and P.Y. Bard. Green functions and associated sources in infinite and stratified poroelastic media. Geophysics J. R. Astr. Soc. Vol. 90, pp. 521-550, 1987

[13] C.W. Holland and B.A. Brunson. The Biot Model: An experimental assessment, J. Acoustical Society of America, Vol. 84, No. 4, pp. 1437-1443, 1988

[14] J. Buchanan and R.P. Gilbert. Transmission loss in the far-field over a one-layer seabed assuming the Biot sediment model. Zamm Vol. 77, No. 2, pp. 121-135, 1997 